\title{
Compreendendo a sífilis congênita a partir do olhar materno
}

\author{
Understanding the congenital syphilis from the maternal look \\ Comprendiendo la sífilis congénita a partir de la mirada materna
}

\section{Martha Helena Teixeira de Souza ${ }^{\mathrm{I}}$, Elisiane Quatrin Beck ${ }^{\mathrm{II}}$}

\begin{abstract}
Resumo: Objetivo: compreender as percepções maternas sobre sífilis congênita e os cuidados de saúde desses recémnascidos. Método: pesquisa descritiva exploratória de caráter qualitativo, realizada em hospital de médio porte, localizado na cidade satélite Paranoá, Distrito Federal, Brasília, no período de março a agosto de 2017. Fizeram parte do estudo 15 mulheres, mães de recém-nascidos portadores de sífilis congênita. Resultados: os dados resultaram em três eixos temáticos: Falhas na realização do pré-natal; Conhecimento das mães em relação à sífilis congênita e sentimentos das mães acerca do diagnóstico de sífilis congênita. Apesar da realização do pré-natal, evidenciaram-se inseguranças, fragilidades e insuficiência de conhecimentos em relação à doença no que se refere ao diagnóstico, tratamento e prevenção. Conclusão: acredita-se que a educação em saúde, com linguagem acessível e melhores estratégias pelos profissionais de saúde a estas gestantes e parceiros com sífilis, pode-se prevenir a sífilis congênita.
\end{abstract}

Descritores: Sífilis; Sífilis Congênita; Pré-natal; Enfermagem obstétrica; Saúde da mulher

\begin{abstract}
Aim: understand the maternal perceptions about congenital syphilis and the health care of these newborns. Method: this is a qualitative research with a descriptive character, developed in a medium-sized hospital, located in the satellite city Paranoa in the Federal District - Brasilia, between March and August of 2017. Fifteen women, mothers of newborns with congenital syphilis, were part of the study. Results: the data resulted in three thematic axes: Failure to perform prenatal care; Knowledge of mothers regarding congenital syphilis and the feelings of mothers about the diagnosisof congenital syphilis. Despite the completion of prenatal care, there were insecurities, weaknesses and insufficient knowledge regarding the disease regarding the diagnosis, treatment and prevention. Conclusion: it is believed that health education, with accessible language and better strategies by health professionals for these pregnant women and partners with syphilis, can prevent congenital syphilis.
\end{abstract}

Descriptors: Syphilis; Congenital Syphilis; Prenatal; Obstetric nursing; Women's health

Resumen: Objetivo: comprenderlas percepciones maternas sobre sífilis congénita y los cuidados de salud de esos recién nacidos. Método: estúdio cualitativos com caracterdescriptivo, desarrollado en Hospital de porte mediano, localizado em la ciudad satélite Paranoá em el Distrito Federal Brasília, entre los meses de marzo y agosto de 2017. Hicieron parte del estúdio 15 mujeres, madres de recién nacidos portadores de sífilis congénita. Resultados: los

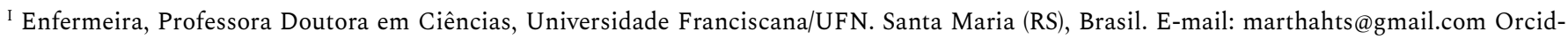
https://orcid.org/0000-0002-5898-9136

II Enfermeira egressa do Mestrado Profissional em Saúde Materno Infantil, Universidade Franciscana /UFN. Santa Maria (RS), Brasil. lisaquatrin@gmail.com; Orcid - https://orcid.org/0000-0003-0138-4248
} 
datos resultaron em tres ejes temáticos: Fallas em La realización del prenatal; Conocimiento de las madres en relación a la sífilis congénita y Sentimientos de las madres sobre el diagnóstico de sífilis congénita. A pesar de la finalización de la atención prenatal, hubo inseguridades, debilidades y conocimiento insuficiente sobre la enfermedad en términos de diagnóstico, tratamiento y prevención. Conclusión: se cree que la educación em salud, com lenguaje accesible y mejores estratégias por los profesionales de salud a estas gestantes con sífilis, puede prevenir la sífilis congénita.

Descriptores: Sífilis; Sífilis Congénita; Prenatal; Enfermería Obstétrica; Salud de la Mujer

\section{Introdução}

A sífilis é uma doença infecciosa sistêmica de evolução crônica, causada pela bactéria gram-negativa Treponema pallidum, do grupo das espiroquetas de alta patogenicidade. A história natural da doença é caracterizada por fases de atividade clínica e de latências de transmissão predominantemente sexual. ${ }^{1}$

Tem sido observado um aumento da prevalência da sífilis em países em desenvolvimento e industrializados, destacando-se o aumento dos casos de sífilis primária e secundária em mulheres em idade fértil. De acordo com o Boletim de Vigilância Epidemiológica no período de 2005 a junho de 2018, foram notificados no Sistema de Informação de Agravos de Notificação (SINAN) 259.087 casos de sífilis em gestantes. ${ }^{2}$ A sífilis na gestação leva a mais de 300 mil mortes fetais e neonatais por ano no mundo, e coloca um adicional de 215 mil crianças em aumento do risco de morte prematura. ${ }^{3}$

Já em relação à sífilis congênita de 1998 a junho de 2018, foram notificados no Sinan 188.445 casos de sífilis congênita em menores de um ano de idade, dos quais $83.800(44,5 \%)$ eram residente na Região Sudeste, $57.422(30,5 \%)$ no Nordeste, $20.922(11,1 \%)$ no Sul, $15.898(8,4 \%)$ no Norte e 10.403 (5,5\%) no Centro Oeste. ${ }^{2}$ Esses números demonstram aumento dos registros de Sífilis Congênita (SC), o que sugere dificuldades no diagnóstico e/ou na notificação desse agravo e deficiências na qualidade da assistência ao pré-natal e ao parto. ${ }^{4}$ Esses fatores resultam em alto custo financeiro para o sistema de saúde, além de um impacto social relacionado às sequelas da doença. ${ }^{5}$ 
Diante disso, é preciso conhecer a Rede de Atenção à Saúde (RAS), ou seja, o território onde as pessoas vivem os serviços de saúde existentes, o fluxo de atendimento, o processo de trabalho dos profissionais envolvidos no cuidado, as características epidemiológicas e socioeconômicas locais. Isso constitui um instrumento essencial para qualificar a adoção de políticas públicas de saúde. ${ }^{5}$

É de grande relevância analisar a compreensão e a percepção das mulheres que vivenciaram a experiência de ter filhos com SC, de modo que o conteúdo e as representações simbólicas que permeiam as atitudes e práticas dessas mulheres possam melhorar a compreensão do cuidado com a doença. Além disso, considera-se importante intensificar as pesquisas sobre esse aspecto do contexto da SC, visto que os estudos existentes se concentram em dados epidemiológicos, o que é compreensível diante da gravidade desta doença. Sob essa perspectiva, destaca-se a questão que norteou a presente proposta investigativa: como mães de recém-nascidos com SC compreendem os cuidados com essa doença? Tendo em vista o exposto, o presente artigo teve como objetivo compreender as percepções maternas sobre SC e os cuidados de saúde desses recém-nascidos com a doença.

\section{Método}

Trata-se de pesquisa qualitativa com caráter descritivo. A pesquisa qualitativa aborda questões que não podem ser quantificadas, buscando compreender valores e significados de fenômenos humanos. ${ }^{6}$ A pesquisa foi desenvolvida em hospital de médio porte, localizado na cidade satélite Paranoá, Distrito Federal, Brasília. O local para a realização do estudo foi escolhido por ser o ambiente de trabalho da pesquisadora, no qual vem sendo observado um aumento de casos de SC nos últimos anos. Responderam os questionamentos 15 mulheres, mães de recém-nascidos com SC. Foram critérios de inclusão na amostra: ter mais de 18 anos e estar 
internada acompanhando o tratamento de seu filho. Como critérios de exclusão, considerou-se: residir fora da área de abrangência do estudo.

Realizou-se entrevista individual com as participantes, entre os meses de março a agosto de 2017. As questões norteadoras foram transcritas, baseadas nos seguintes questionamentos: Fale o que você sabe sobre sífilis e sífilis congênita. Em que momento recebeu o resultado positivo para sífilis? Onde seu teste foi realizado e como procedeu a partir desse momento? Quantas consultas você realizou durante o pré-natal? Como realizou o tratamento? O seu parceiro realizou tratamento no serviço de saúde?

As entrevistas foram realizadas respeitando a privacidade e interação entre pesquisadora e participantes. O local também favoreceu a contextualização de experiências, vivências, sentidos que contribuíram para esclarecer a problemática da investigação. Os dados foram coletados após aprovação do projeto no Comitê de Ética em Pesquisa, da Universidade Franciscana - UFN, sob o parecer nº 1.881.757. A identificação das participantes realizou-se a partir das iniciais MI (Mulheres Internadas) de acordo com a entrada no texto (MI1, MI2,..., MI15).

Os resultados foram analisados preconizando os seguintes passos: a ordenação dos dados coletados, a classificação dos dados e a análise final (levando em conta os objetivos da pesquisa e os temas que emergiram das entrevistas). ${ }^{7}$ Após a concretização desse processo, apresenta-se uma discussão dos achados, a aproximação com a literatura já existente e pertinente à temática.

\section{Resultados e discussões}

Participaram deste estudo mães de recém-nascidos com SC com idades entre 18 a 37 anos, sendo que a quase totalidade não havia completado o ensino fundamental. A maioria das entrevistadas com renda familiar de um salário mínimo e ausência de ocupação remunerada. Algumas declararam possuir apenas o benefício social "Bolsa Família”, o qual consiste no 
repasse de recursos financeiros do governo diretamente para famílias em situação de pobreza, mediante cumprimento das condicionalidades em educação em saúde. Todas as entrevistadas eram multíparas e mantinham contato ativo com seu parceiro sexual.

Com relação às condições sociais das entrevistadas percebe-se que possuíam baixa escolaridade. Esses dados foram semelhantes à pesquisa desenvolvida em Sobral, município de médio porte do nordeste brasileiro, cuja maior predominância foi em mães pardas e com ensino fundamental incompleto. ${ }^{8}$

Frequentemente, este é o perfil de indivíduos com uma condição socioeconômica menos favorecida e com menos acesso à saúde de qualidade. Contudo, não se pode afirmar que a sífilis seja uma condição de risco exclusivamente de populações mais carentes, ao contrário, independentemente da condição social ou econômica, todos podem adquirir a infecção, porém, o risco é maior em populações mais vulneráveis. ${ }^{9}$

\section{Falhas na realização do pré-natal}

A maioria das entrevistadas informou que a realização de pré-natal ocorreu no período adequado, ou seja, no primeiro trimestre da gestação. Apenas uma das mulheres mencionou ter iniciado tardiamente no terceiro trimestre de gestação. Ao narrarem o momento do diagnóstico, revelaram que este foi feito na primeira consulta, por meio do teste rápido para sífilis.

Apesar de haver maior oferta de testes de diagnósticos para as gestantes e seus parceiros, com a introdução dos testes rápidos nas Unidades de Saúde, os dados epidemiológicos não demonstraram declínio de casos. Esse fato evidencia que somente o acesso ao diagnóstico não é suficiente para melhoria da qualidade de atenção à gestante portadora de sífilis. ${ }^{10}$

Salienta-se que cinco das entrevistadas tiveram aborto e duas, natimorto. Destas, sete receberam o diagnóstico de sífilis em gestações anteriores. Evidenciaram-se, neste estudo, seis partos prematuros e nove partos termos, que poderiam ou não estar associados à SC. É sabido 
que a contaminação do feto pode resultar em aborto, natimorto, prematuridade ou um amplo espectro de manifestações clínicas; apenas os casos muito graves são clinicamente aparentes ao nascimento. De acordo com Ministério da Saúde entre mulheres com sífilis precoce não tratada, $40 \%$ das gestações resultam em aborto espontâneo. ${ }^{11}$

Em relação ao tratamento, nove mulheres realizaram três doses da medicação preconizada pelo Ministério da Saúde. No entanto, apenas três parceiros realizaram conjuntamente o tratamento. É constante o dado de que o principal fator de risco para a SC consiste no acompanhamento pré-natal inadequado, relacionado à cerca de 70 a $90 \%$ dos casos. Dentre os problemas relacionados ao atendimento pré-natal, destacam-se sorologia para sífilis não realizada nos períodos preconizados (1ำ e $3^{\text {o }}$ trimestres); interpretação inadequada da sorologia para sífilis; não tratamento do parceiro sexual, informações inadequadas veiculadas entre a equipe de assistência. ${ }^{12}$

Embora os resultados apontem para uma busca adequada pelo pré-natal, não foi assegurado um desfecho favorável do tratamento da sífilis, gerando o diagnóstico de SC no bebê. Uma melhor organização dos serviços de saúde, o monitoramento constante dos casos de sífilis em gestantes e SC juntamente com a sensibilização dos profissionais podem diminuir as falhas na prevenção e assistência aos casos de sífilis. ${ }^{10}$

Observou-se que o principal fator de falha no tratamento das gestantes foi a falta e/ou a inadequação do tratamento do parceiro sexual. Essa é uma das deficiências que permanecem na atenção primária do país, principalmente, no momento do pré-natal. Apesar dos resultados indicarem um aumento considerável na adesão às consultas de pré-natal e, consequentemente, um diagnóstico mais precoce da infecção, o tratamento das gestantes ainda é majoritariamente inadequado e seus parceiros, em sua maioria, não são tratados, contribuindo assim para desfechos indesejáveis. ${ }^{13}$ 
Durante as entrevistas, percebeu-se a falta de orientações a respeito da doença e de sua prevenção:

A doença do meu filho poderia ter sido evitada. Faltou informação sobre a necessidade do uso de preservativo nas relações com meu parceiro, que o não uso acarretaria problemas para meu bebê. Pensei que não teria problema para o bebê, pois tomei as três doses da medicação. (MI5)

Evidencia-se a necessidade de qualificação do atendimento realizado no pré-natal. Tal ação pode acontecer por meio da capacitação dos profissionais de saúde, a fim de auxiliar na identificação precoce de gestantes infectadas, reduzindo o número de casos da doença e seus agravos. ${ }^{14}$ Dessa forma, poderá haver aumento da adesão ao tratamento e redução da vulnerabilidade das mulheres e seus parceiros às Infecções Sexualmente Transmissíveis (ISTs).

\section{Conhecimento das mães em relação à sífilis congênita}

Quando questionadas a respeito da transmissão da sífilis, as mães participantes da pesquisa apontaram o risco da relação sexual desprotegida. No entanto, em relação à transmissão da SC, mostraram-se, por vezes, confusas:

Sei que a sifilis transmite por relação sexual, mas não sei direito como passa para o bebê. Mesmo eu tomando injeções, preciso continuar cuidando?(M14)

Essas questões podem ser esclarecidas durante o pré-natal, uma vez que o momento em que ocorrem essas consultas se constitui em um espaço rico para debater dúvidas e evitar a SC. A consulta de pré-natal quando não é compreendida como um momento de acolhimento, pode diminuir a satisfação e confiança da gestante no profissional que conduz seu atendimento. ${ }^{15}$ As mães afirmaram saber que seus filhos poderiam desenvolver sequelas, caso recebessem o diagnóstico de SC, no entanto demonstraram incertezas ao relatar quais seriam essas 
complicações. As informações sobre as sequelas da SC, algumas vezes, foram adquiridas pela televisão e internet:

Eu vi na televisão que a criança pode nascer surda. (M11)

Vi na internet que causa mancha no corpo, ferida nas partes íntimas. (MI2)

Um problema recorrente, apontado pelas participantes da pesquisa, foi em relação as orientações recebidas durante o atendimento pré-natal por parte dos profissionais de saúde. As falas evidenciam uma comunicação insuficiente:

A médica do pré-natal me informou que o bebê poderia nascer com problemas de coração, cérebro e mal- formação. Mas não entendi direito como seria isso. (MI8)

No posto falaram que era ruim para o bebê, e que tinha que tratar, fiquei com medo. Mas não entendi direito.(MI9)

De acordo com a Organização Mundial de Saúde (OMS), os usuários dos serviços de saúde devem receber informação sobre a sífilis e ser convencidos de que a prevenção e o tratamento podem resultar em benefícios importantes para a saúde materna e da criança que irá nascer. É importante ressaltar que abordagens comunitárias podem ser importantes para informar o público vulnerável e estimular a procura de meios para a detecção da sífilis. ${ }^{16}$

Em relação ao tratamento, observaram-se novas dúvidas:

Eu ainda não sei muitas coisas, me disseram, mas eu não entendi muito. Parece que duram 10 dias. (MI9)

Não sei direito sobre o tratamento. Não me explicaram, [...] estou tomando umas injeções. (MI10)

A complexidade do tratamento para SC, somada ao desconhecimento sobre a doença, pode explicar as limitações das puérperas quanto à compreensão da terapêutica adequada. No entanto, percebeu-se, por meio dos depoimentos, a necessidade de uma melhor orientação, pois o tratamento correto consiste em chance de cura para o recém-nascido $(\mathrm{RN})$ e prevenção de complicações graves. 
Salienta-se que, após a alta, a criança deverá permanecer em acompanhamento ambulatorial mensal até os seis meses de vida e bimestrais do sexto ao $12^{\circ}$ mês. A realização de exames não treponêmicos deve ser regular até 18 meses ou dois exames consecutivos negativos. O acompanhamento especializado em oftalmologia, neurologia e audiologia devem ser semestrais até os dois anos de vida. ${ }^{17} \mathrm{~A}$ insegurança das mães em relação à SC pode gerar diferentes desconfortos, os quais talvez venham a intervir no seguimento do tratamento das crianças infectadas.

\section{Sentimentos das mães acerca do diagnóstico de Sífilis Congênita}

Durante os depoimentos, as mães expressaram-se com emoção ao recordarem-se do momento do diagnóstico da doença. Nesse sentido, narraram alguns sentimentos de angústia e preocupação em relação à infecção dos filhos:

Fiquei muito chateada quando recebi o diagnóstico, porque a criança não tem culpa de nada. Sinto muita tristeza, chorei muito. Eu não gosto porque ele chora, eu queria sentir a dor dele, o que ele está sentindo eu queria sentir. Eu não gosto de jeito nenhum. (MI3)

Eu deveria ter convencido meu marido a fazer o tratamento, assim nosso filho não teria nascido com essa doença. Fico triste. Sabendo que ela pegou de mim, mãe nenhuma quer ver seu filho sofrer. (MI5)

É horrível. Quando via o bebê sendo picado e saber que a culpa foi nossa. Depois que vi meu filho internado percebi que o tratamento é importante. É uma situação muito difícil para mim, porque ver meu filho chorando cada vez que ele toma essas injeções, me faz sofrer, porque pegou de mim. (MI2)

As narrativas revelaram culpa e sofrimento. Fatores como sentimento de remorso e falta de informações parecem influenciar sua compreensão em relação ao tratamento, podendo comprometer o acompanhamento do RN. O apoio a essas mulheres é muito importante, assim como não permitir que haja o repasse de culpa pela transmissão da doença para elas. Ao invés 
de reforçar sua culpa sobre a responsabilidade na circunstância em que vive, o profissional deverá, sim, adotar um diálogo franco, com uma linguagem sucinta.

Diversos sentimentos de inquietação e insegurança podem ser reduzidos por meio de uma comunicação clara, eficaz e pontual por parte da equipe, tornando o vínculo mais sólido e efetivo. ${ }^{18}$ A equipe de saúde, nesse contexto, têm um papel fundamental atuando como mediadores das necessidades desse contingente populacional, oferecendo suporte assistencial, orientações e esclarecimentos.

Assim, de modo individual ou em grupo, com a adoção de estratégias educativas e respeitando as crenças e a cultura de cada mulher, esses trabalhadores podem contribuir de modo expressivo para a adesão ao exame. Além disso podem conscientizá-las sobre a importância da realização do exame preventivo e incentivar a procura pelas unidades de saúde. ${ }^{19-20}$

Os elementos, que permeiam o diagnóstico, o tratamento e a prevenção da sífilis e SC em mulheres, poderão sinalizar fatores que estejam influenciando o cuidado e, dessa forma, favorecer a implementação de estratégias de educação em saúde que visem à saúde dessa população. Esse método pode incluir o trabalho de educação em saúde utilizando materiais informativos e estar atenta ao que a usuária do serviço compreendeu da mensagem.

\section{Considerações finais}

O estudo mostrou a importância de revisão dos procedimentos adotados e maior percepção dos profissionais de saúde e gestores perante a SC principalmente, nos aspectos relativos à comunicação com as usuárias do serviço. Tendo em vista as falhas percebidas no cuidado dessas mulheres durante a realização do seu pré-natal. Evidenciado, ao longo da pesquisa, inseguranças em relação ao diagnóstico, ao tratamento e à prevenção.

O conhecimento das mulheres é fundamental para que haja adesão ao tratamento da doença e a prevenção adequada. Sugere-se a promoção de ações educativas por meio de 


\section{I Souza MHT, Beck EQ}

materiais informativos, de linguagem acessível, que sejam distribuídos às mães no momento da alta, para o seguimento dos cuidados com seu RN. No caso da sífilis adquirida e da SC, a prevenção já é conhecida e disseminada no ambiente da saúde, porém, são necessárias melhores estratégias para colocar em prática a prevenção.

Entretanto, uma limitação deste estudo foi a não participação dos parceiros na pesquisa, visto que inicialmente havia a previsão de participação das mulheres, mães de RN com SC. Destaca-se que incluir os parceiros nesse processo pode elucidar alguns pontos em relação a não realização de tratamento efetivo nos mesmos.

A SC é uma condição evitável, desde que corretamente diagnosticada e tratada. A persistência de altas taxas de transmissão vertical, mesmo após a busca por assistência ao prénatal, indica dificuldades para o seu controle e intervenção. A fragilidade no diagnóstico e tratamento da mulher e do parceiro amplia a vulnerabilidade para a ocorrência de SC. Portanto, acredita-se que a orientação de um profissional de saúde às gestantes com sífilis, principalmente, sobre os riscos que as crianças correm ao se infectarem com o Treponema pallidum, podem minimizar os riscos da SC.

\section{Referências}

1. Secretaria de Estado da Saúde [do Estado de São Paulo], Centro de Controle de Doenças. Programa Estadual de DST/Aids. Guia de bolso para o manejo da sífilis em gestante e sífilis congênita. São Paulo (SP): Secretaria de Estado da Saúde; 2016. 112 p.

2. Ministério da Saúde (BR), Secretaria de Vigilância em Saúde. Boletim epidemiológico: sífilis. 2018;49 (45).

3. Ministério da Saúde (BR), Secretaria de Vigilância em Saúde. Boletim epidemiológico: sífilis. 2016;47 (47).

4. Domingues RMSM, Szwarcwald CL, Souza Junior PRB, Leal MC. Prevalência de sífilis na gestação e testagem pré-natal: estudo nascer no Brasil. Rev Saúde Pública. 2014;48(5):766-74.

5. Sortica AC. Rede de atenção à saúde, sífilis e educação em saúde, a intersecção necessária: um estudo de caso sobre sífilis em gestante e congênita no município de Esteio [dissertação]. Porto Alegre: Universidade Federal do Rio Grande do Sul; 2017. 
6. Minayo MCS. Pesquisa Social: teoria, método e criatividade. 34를 ed. Petrópolis: Vozes; 2015.

7. Minayo MCS. O desafio do conhecimento: pesquisa qualitativa em saúde. $14^{a}$ ed. São Paulo (SP): Hucitec; 2014.

8. Lima VC, Mororó RM, Martins MA, Ribeiro SM, Linhares MSC. Perfil epidemiológico dos casos de sífilis congênita em um município de médio porte no nordeste brasileiro. J Health Biol Sci. 2017;5(1):56-61.

9. Rufino EC, Andrade SSC, Leadebal ODCP, Brito KKG, Silva FMC, Santos SH.Conhecimento de mulheres sobre ist/aids: intervindo com educação em saúde/ Women's knowledge about sti/aids: working with health education. Cienc Cuid Saúde [Internet]. 2016 [acesso em 2018 mar 12];15(2):304-11. Disponível em: http://periodicos.uem.br/ojs/index.php/CiencCuidSaude/article/view/26287

10. Saraceni V, Pereira GFM, Silveira MF, Araújo MAL, Miranda AE. Vigilância epidemiológica da transmissão vertical da sífilis: dados de seis unidades federativas no Brasil. Rev Panam Salud Publica, Washington. 2017;41(44).

11. Ministério da Saúde (BR). Protocolo clínico e diretrizes terapêuticas para prevenção da transmissão vertical de HIV, sífilis e hepatites virais. Brasília (DF): Ministério da Saúde; 2019. p. 183.

12. Ministério da Saúde (BR). Protocolo clínico e diretrizes terapêuticas. Prevenção da transmissão vertical de HIV, sífilis e hepatites virais: CONITEC, 2017 [acesso em 2018 mar 12]. Disponível em: http://www.aids.gov.br/pt-br/pub/2015/protocoloclinico-e-diretrizes-terapeuticas-para-prevencaodatransmissao-vertical-de-hiv

13. França ISX, Batista JDL, Coura AS, Oliveira CF, Araújo AKF, Sousa FS. Fatores associados à notificação da sífilis congênita: um indicador de qualidade da assistência pré-natal. Rev Rene. 2015 maiojun; 16(3):374-81.

14. Rodrigues VLR, Oliveira FM, Afonso TM. Sífilis congênita na perspectiva de um desafio para a saúde pública. Congresso internacional de enfermagem; 2017; Aracajú, SE. Anais. (CIE Unit. 2017 [acesso em 2018 abr 14];1(1). Disponível em: https://bit.ly/2zE1A2C

15. Andrade FM, Castro JFL, Silva AV. Percepção das gestantes sobre as consultas médicas e de enfermagem no pré-natal de baixo risco. Rev Enferm Cent Oeste Min. 2016 set-dez;6(3):2377-88.

16. Lima VC, Mororó RM, Feijão DM, Frota MVV, Martins MA, Ribeiro SM, et al. Percepção de mães acerca da sífilis congênita em seu concepto. Espaço para a saúde. Rev Saúde Pública Paraná. 2016. 17(a2):118-25.

17. Ministério da Saúde (BR), Secretaria de Vigilância em Saúde. Programa Nacional de DST e Aids e hepatites virais. Protocolo clínico e diretrizes terapêuticas para atenção integral às pessoas com infecções sexualmente transmissíveis. Brasília (DF): Ministério da Saúde; 2015. 
18. Godinho MLM, Dias MV, Barlem ELD, Barlem JGT, Rocha LP, Ferreira AG. Diretivas antecipadas de vontade: percepção acerca da aplicabilidade no contexto neonatal e pediátrico. Rev Enferm UFSM 2018 jul-set [acesso em 2018 fev 05];8(3):475-88. Disponível em: https://periodicos.ufsm.br/reufsm/article/view/27887

19. Santos LV, Inagaki ADM, Abud ACF, Oliveira JKA, Ribeiro CJN, Oliveira MIA. Características sociodemográficas e risco para doenças sexualmente transmissíveis entre mulheres atendidas na atenção básica. Rev Enferm UERJ [Internet]. 2014 [acesso em 2018 fev 05]; 22(1):111-5.Disponível em: http://www.facenf.uerj.br/v22n1/v22n1a17.pdf

20. Oliveira AEC, Deininger LSC, Lima IMB, Lima DC, Nascimento JA, Andrade JM. Adesão das mulheres ao exame citológico do colo uterino na atenção básica. Rev Enferm UFPE On Line [Internet]. 2016 [acesso em 2018 fev 05];10(11):4003-14. Disponível em: http://pesquisa.bvsalud.org/enfermeria/resource/pt/bde-3014

\section{Autor correspondente}

Elisiane Quatrin Beck

E-mail: lisaquatrin@gmail.com

Endereço: Rua Victorino da Cás nº60 casa 31B Bairro Cerrito Santa Maria RS

CEP: 97060491

\section{Contribuições de Autoria}

\section{1 - Martha Helena Teixeira de Souza}

Orientadora da pesquisa, concepção e planejamento do projeto de pesquisa, análise e interpretação dos dados, redação e revisão crítica.

\section{2 - Elisiane Quatrin Beck}

Pesquisadora responsável pela coleta de dados, concepção e planejamento do projeto de pesquisa, análise e interpretação dos dados, redação e revisão crítica.

\section{Como citar este artigo}

Souza MHT, Beck EQ. Compreendendo a sífilis congênita a partir do olhar materno. Rev. Enferm. UFSM. 2019 [Acesso em: Anos Mês Dia];vol 9; e56: 1-13. DOI:https://doi.org/10.5902/217976932072 